NASA Contractor Report 191200

AIAA-94-0917

\title{
A Laboratory System for the Investigation of Rain Fade Compensation Techniques for Ka-Band Satellites
}

James S. Svoboda

Sverdrup Technology, Inc.

Lewis Research Center Group

Brook Park, Ohio

and

Brian A. Kachmar

Analex Corporation

Brook Park, Ohio

Prepared for

Lewis Research Center

Under Contracts NAS3-25266 and NAS3-25776

\section{N/SA}




\title{
A LABORATORY SYSTEM FOR THE INVESTIGATION OF RAIN FADE COMPENSATION TECHNIQUES FOR Ka-BAND SATELLITES
}

\author{
James S. Svoboda \\ Sverdrup Technology, Inc. \\ Lewis Research Center Group \\ Brook Park, Ohio 44142 \\ and \\ Brian A. Kachmar \\ Analex Corporation \\ Brook Park, Ohio 44142
}

\begin{abstract}
$\underline{\text { Abstract }}$
The design and performance of a rain fade simulation/ counteraction system on a laboratory simulated $30 / 20 \mathrm{GHz}$, time division multiple access (TDMA) satellite communications testbed is evaluated. Severe rain attenuation of electromagnetic radiation at $30 / 20 \mathrm{GHz}$ occurs due to the carrier wavelength approaching the water droplet size. Rain in the downlink path lowers the signal power present at the receiver, resulting in a higher number of bit errors induced in the digital ground terminal. The laboratory simulation performed at NASA Lewis Research Center uses a programmable PIN diode attenuator to simulate $20 \mathrm{GHz}$ satellite downlink geographic rain fade profiles. A computer based network control system monitors the downlink power and informs the network of any power threshold violations, which then prompts the network to issue commands that temporarily increase the gain of the satellite based traveling wave tube (TWT) amplifier. After the rain subsides, the network returns the TWT to the normal energy conserving power mode. Bit error rate (BER) data taken at the receiving ground terminal serves as a measure of the severity of rain degradation, and also evaluates the extent to which the network can improve the faded channel.
\end{abstract}

\section{Introduction}

Modern communication satellite designs employ many techniques aimed at increasing the efficiency of data throughput. Raising the carrier frequencies and increasing spectral bandwidth is one technique implemented in the design of modern communication satellites, such as in NASA's Advanced Communications Technology Satellite (ACTS), that increases data throughput but creates additional design considerations. Raising the uplink and downlink carrier frequencies to a less congested frequency band enables greater data throughput since more spectral bandwidth is available at these higher frequencies. This increase in data throughput does not come without a price, and many system design tradeoffs and problems must be considered. One major problem deals with the transmission of electromagnetic RFenergy athigh frequencies which creates additional fading problems that can lower the received power to unacceptable levels.
RF energy at $30 / 20 \mathrm{GHz}$ experiences atmospheric losses much greater than signals transmitted through the atmosphere at lower frequencies. Water vapor and oxygen present in the atmosphere attenuates, scatters, and radiates electromagnetic energy at different frequency dependent levels. Water vapor is particularly detrimental to the $30 / 20 \mathrm{GHz}$ band, and this problem is further compounded when rain resides in the transmission path.

Satellite TWTs could operate continuously at high power levels to provide a wide enough link margin to overpower any rain fade likely to occur. This method, however, wastes expensive satellite power, and contributes to intersystem interference and is therefore not considered a practical solution. Other methods proposed to combat rain fade on a satellite downlink path either invoke a form of digital data coding, use adaptive modulation switching techniques, or employ site diversity. Coding requires the transmission of redundant bits which adds to the already large amount of data transmitted in a limited frequency band. Adaptive modulation switching responds to fading by switching between various M-PSK modulation schemes, each at a different data throughput rate. ${ }^{1}$ The problem with these compensation methods is that they require more bandwidth, or a reduced data throughput rate, to achieve an improvement in signal to noise ratio, which may not be an acceptable sacrifice in an already crowded frequency band. The drawback of site diversity is the additional hardware required due to the redundant systems. Yet another scheme proposed to lessen the effects of rain employs a hybrid transmitter capable of switching to a lower and less rain susceptible frequency band during a rain fade. The disadvantage of this method, besides the increase in the amount of hardware, is again the fact that users must tolerate lower data throughput or wider bandwidth requirements during a rain fade event.

The method evaluated in this study to eliminate rain induced errors uses an adaptive power control scheme that maintains constant bandwidth and a constant data rate while combating rain fade through downlink power control. A multigain (three power mode) TWT amplifier overpowers rain by changing power modes in response to telemetry 




Figure 1.-System diagram.

commands transmitted by the ground terminal. The telemetry commands originate from a downlink power sensing system that monitors the received power prior to the demodulator. If power drops below a certain threshold, commands transmitted to the satellite temporarily change the TWT to a higher power mode. The test ground terminal continuously transmits data through the channel while counting the number of bits in error, thus producing a BER figure.

The main goal of this study is to design and characterize a laboratory simulated satellite channel to prove the feasibility and evaluate the performance of a multipower mode rain fade control technique. As shown in Fig. 1, rain is simulated in the $20 \mathrm{GHz}$ downlink path by a programmable PIN diode attenuator driven by computer controlled geographic fade prediction models based on location specific rainfall statistics. Power variations sensed at the input to the test ground terminal are then combatted via temporary TWT power mode changes. During clear sky transmission, the TWT operates in the lowest power mode capable of maintaining an acceptable link bit error rate. Several different TDMA burst data rates have been tested to identify any component sensitivity due to a change in burst density. Empirical BER data plotted versus energy per bit per noise power density ratio $\left(\mathrm{E}_{\mathrm{b}} / \mathrm{N}_{0}\right)$ is the major means of data comparison indicating the severity of the rain fade and the amount of improvement the power control scheme provides.

\section{Laboratory Transponder Configuration}

Higher frequency bands offer new opportunities for satellite system designers, including less crowding, less interference, and greater spectral bandwidth. To aid in the transition to higher carrier frequencies, NASA Lewis has established a $30 / 20 \mathrm{GHz}$ satellite communications network facility known as the Advanced Space Communications Laboratory (ASCL). This facility was originally created due to NASA's research involvement in the development and evaluation of proof-ofconcept (POC) components for the ACTS $30 / 20 \mathrm{GHz}$ communications satellite, and the need for a technology verification testbed. The ASCL facility characterized such POC components as satellite based matrix switches, TWT amplifiers, low noise $30 \mathrm{GHz}$ satellite receivers, digital ground terminal architectures, modems, and solid state amplifiers for the ACTS project.

The present configuration of the ASCL network simulator is a satellite switched time division multiple access (SS-TDMA) network. The network will ultimately demonstrate a three ground terminal configuration where each ground terminal can simulate satellite acquisition and maintain network synchronization while transmitting/receiving bursted TDMA data between other ground terminals. One will serve as a Master Control Terminal (MCT) and be responsible for communication 


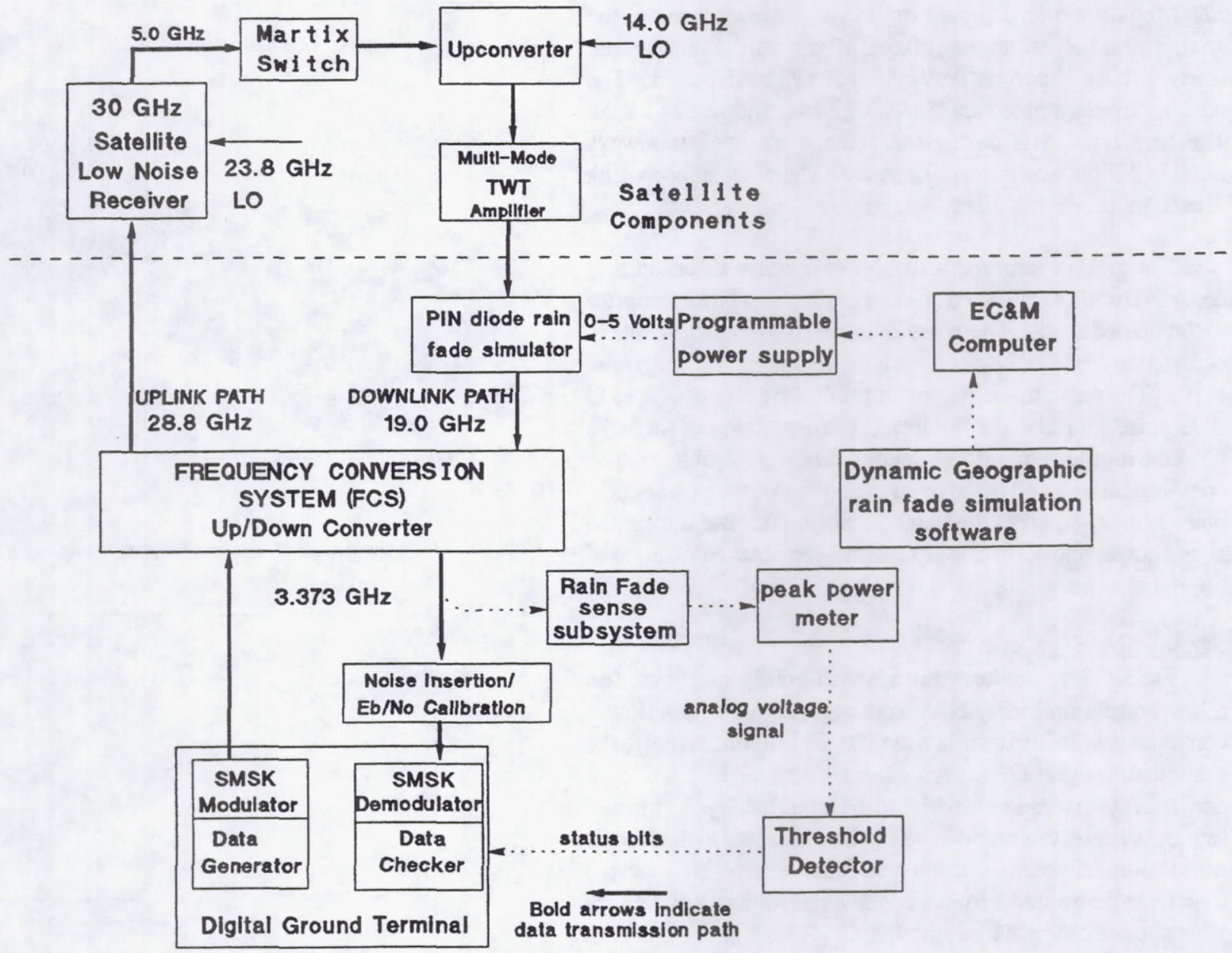

Figure 2.-Rain fade simulation/counteraction system.

with the Network Control Computer (NCC). The NCC services requests for access to the network, manages all users, configures the matrix switch, and controls satellite TWT power. ${ }^{2}$ A more detailed description of the computer control system will be presented later. The following section will describe the major components in the laboratory transponder based on Fig. 2 .

\section{Digital Ground Terminal}

The NASA Lewis designed and built digital ground terminal is the key piece of test equipment used in the ASCL for the evaluation of satellite components via its bit error rate measurement capability. The heart of the digital ground terminal is the data generator and data checker pair. The data generator creates a 221 Mega bitpersecond(Mbps) pseudorandom data sequence with a bit pattern that repeats every 65536 words, with 64 bits per word. ${ }^{3}$ The data pattern is then routed to the Motorola serial minimum shift keyed (SMSK) modem. The modem has an output center frequency of $3.373 \mathrm{GHz}$ and a main lobe spectral bandwidth of approximately $330 \mathrm{MHz}$, which is 1.5 times the datarate. A spectrum analyzerplot of a continuous (100 percent burst density) $221 \mathrm{Mbps}$ power spectrum is shown in Fig. 3. SMSK has the same BER characteristics as the more popular satellite modulation scheme known as quadrature phase shift keying (QPSK), but is more spectrally efficient. ${ }^{3}$
The digital ground terminal design enables transmission of data at several different duty cycles, or burst densities. In a TDMA system, each user bursts uplink data at a particular time for a period of time dependent on each user's data volume requirements. The test ground terminal produces data bursts at

Ref $0.0 \mathrm{dBm}$

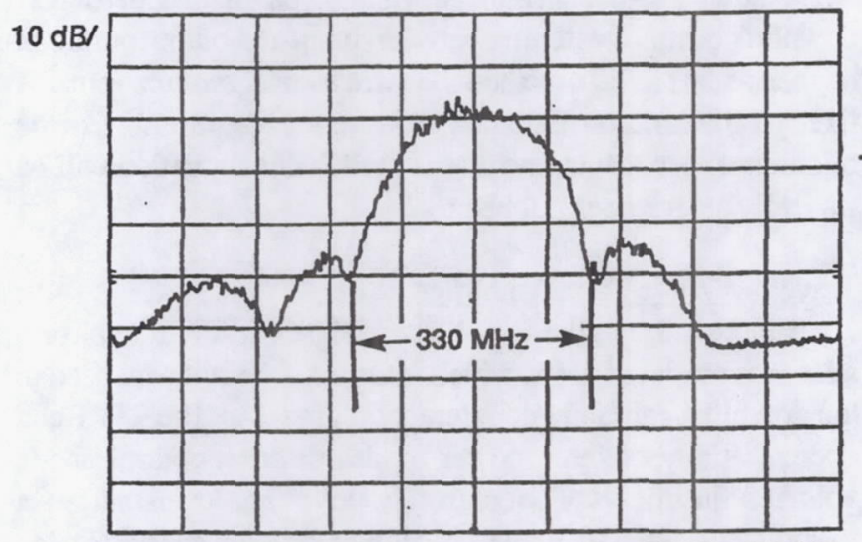

Center $3.37 \mathrm{GHz}$

SPAN $1.00 \mathrm{GHz}$

Figure 3.-220 Mbps SMSK spectrum. 
$221 \mathrm{Mbps}$ for four different duty cycles. For example, the ground terminal can burstat $110.592 \mathrm{Mbps}$ total data throughput rate by using a 50 percent duty cycle of its $221 \mathrm{MHz}$ clock. The other data rates utilized are: $55.296,27.648$, and $13.824 \mathrm{Mbps}$. The density of data during the actual "on time" is always 221.184 Mbps, but the length of each data burst varies according to each particular burst rate.

The ground terminal also houses a noise insertion unit designed to add a calibrated amount of filtered white noise prior to the demodulator. The noise is added in $1 \mathrm{~dB}$ increments in order to vary the $\mathrm{E}_{\mathrm{b}} / \mathrm{N}_{0}$ to create $\mathrm{BER}$ versus $\mathrm{E}_{\mathrm{b}} / \mathrm{N}_{0}$ "waterfall" curve. The noise insertion unit amplifies the output of a solid state noise source and combines incremental amounts of this power with the actual data signal in a power combiner. The combined signal and noise from the output of the power combiner is then routed to the demodulator input. Measuring the signal power and noise power separately enables calculation of the signal to noise ratio.

\section{Satellite Receiver}

The 30GHz satellite receiver is an in-house design based on a low noise amplifier (LNA) front end followed by a downconverter designed to translate the $28.8 \mathrm{GHz}$ uplink signal to the satellite IF of $5.0 \mathrm{GHz}$. An external $23.8 \mathrm{GHz}$ fixed local oscillator (LO) supplies the drive to the mixer'sLOport. In a real satellite system, this receiver would be connected to the feed of a parabolic dish antenna. In the ASCL, the receiver is connected directly to the ground terminal upconverter followed by a rotary vane attenuator space loss simulator.

\section{Matrix Switch}

The matrix switch is a vital component in a TDMA satellite system employing multiple spot beams rather than a single CONUS beam. The matrix switch directs any one of 20 inputs to any or all of the 20 possible outputs. This capability enables ground terminals in different geographic locations to be connected to one another without involving other unnecessary ground terminals. In other words, when a matrix switch connection is made, the information transmitted is only routed to the receiving ground terminals that need that specific information, while other ground terminals will not be in that particular downlink beam. Switching between input and output ports can happen as fast as $20 \mathrm{nsec}$ and the switch configuration remains fixed for the duration necessary to pass burstlength used. For the rain fade experiments, the matrix switch configuration will be statically set for one spot beam.

\section{Traveling Wave Tube Amplifier}

Figure 4 shows the satellite based $20 \mathrm{GHz}$ TWT amplifier, a Hughes model 918HA, which is the main component used in the downlink rain fade counteraction process. The TWT can operate at any of three power modes through control anode voltage adjustments. The control anode voltage levels can be set to application specific values by tuning variable resistors in the power processing unit (PPU). The PPU is a high voltage power supply consisting of telemetry data meters and computer control interfaces needed by the TWT. Figure 5 shows the present

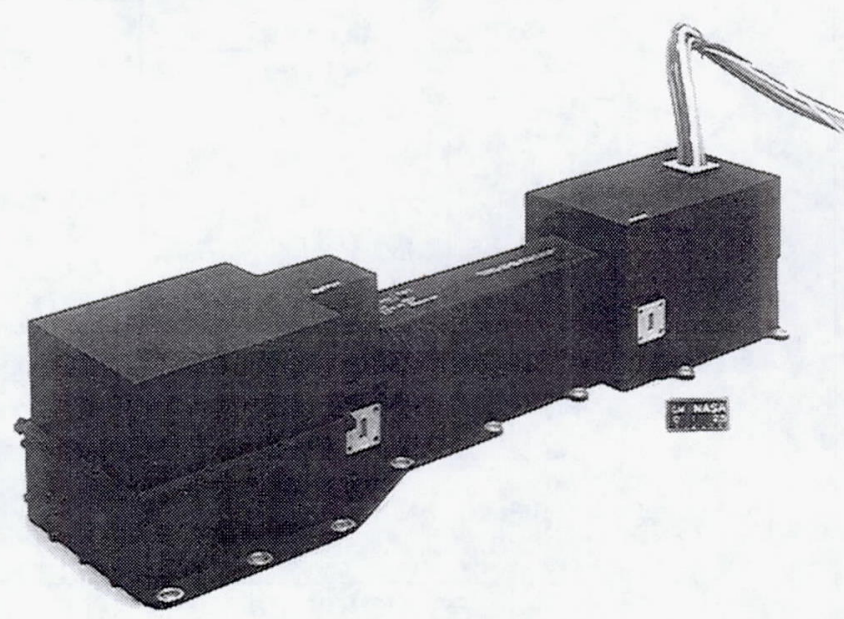

C-\$9-06518

Figure 4.-Hughes model $918 \mathrm{HA}$ multimode traveling wave tube.

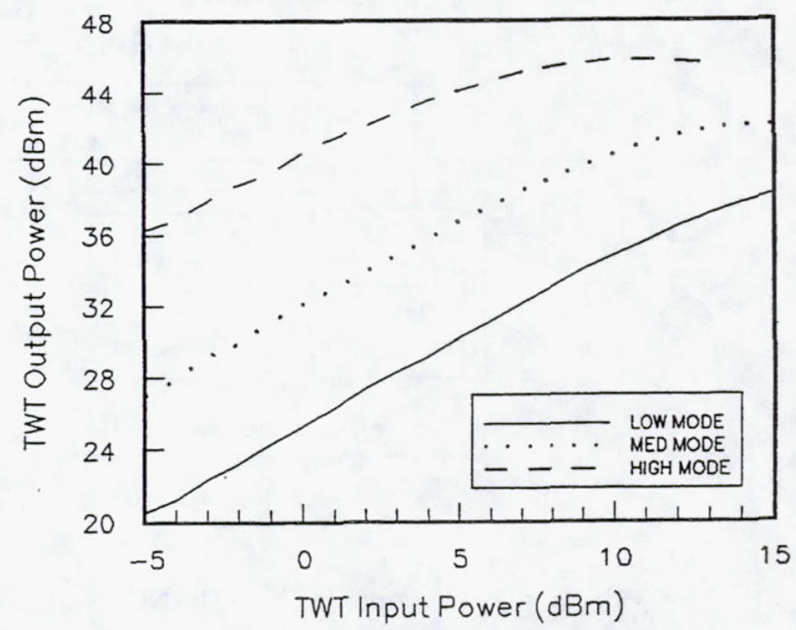

Figure 5.-TWT power curves.

settings for each TWT power mode. The difference in gain between each power mode is approximately $4 \mathrm{~dB}$ between saturation points, and about $8 \mathrm{~dB}$ total difference between low mode saturation and high mode saturation. The design of the TWT limits the maximum possible output to $50 \mathrm{~W}$ of $\mathrm{RF}$ downlink power.

\section{PIN Diode Rain Fade Simulator}

The PIN attenuator shown at the right in Fig. 6, simulates dynamic rain fades in the satellite downlink path. This semiconductor based waveguide device has an attenuation range of 0 to $28 \mathrm{~dB}$ via a control voltage input range of 0 to $5 \mathrm{~V}$. This type of attenuator was chosen over more conventional attenuators due to controlling ease and fastswitching speed. The control voltage is supplied by a programmable power supply and controlled over an IEEE 488 bus that is controlled by the EC\&M computer.

\section{Dynamic Geographic Rain Fade Prediction Model}

NASA Lewis developed a method to simulate dynamic rain fade scenarios using log normal stochastic rain fade prediction models, based on U.S Weather Bureau long term rainfall statis- 


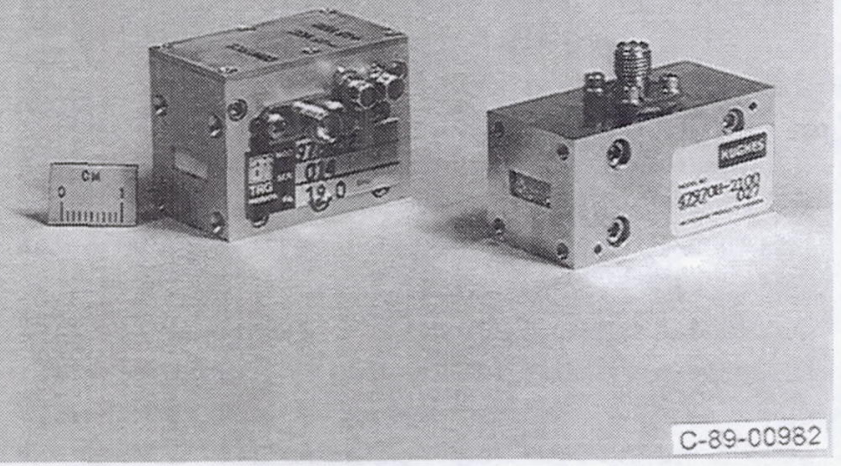

Figure 6.-PIN diode attenuator.
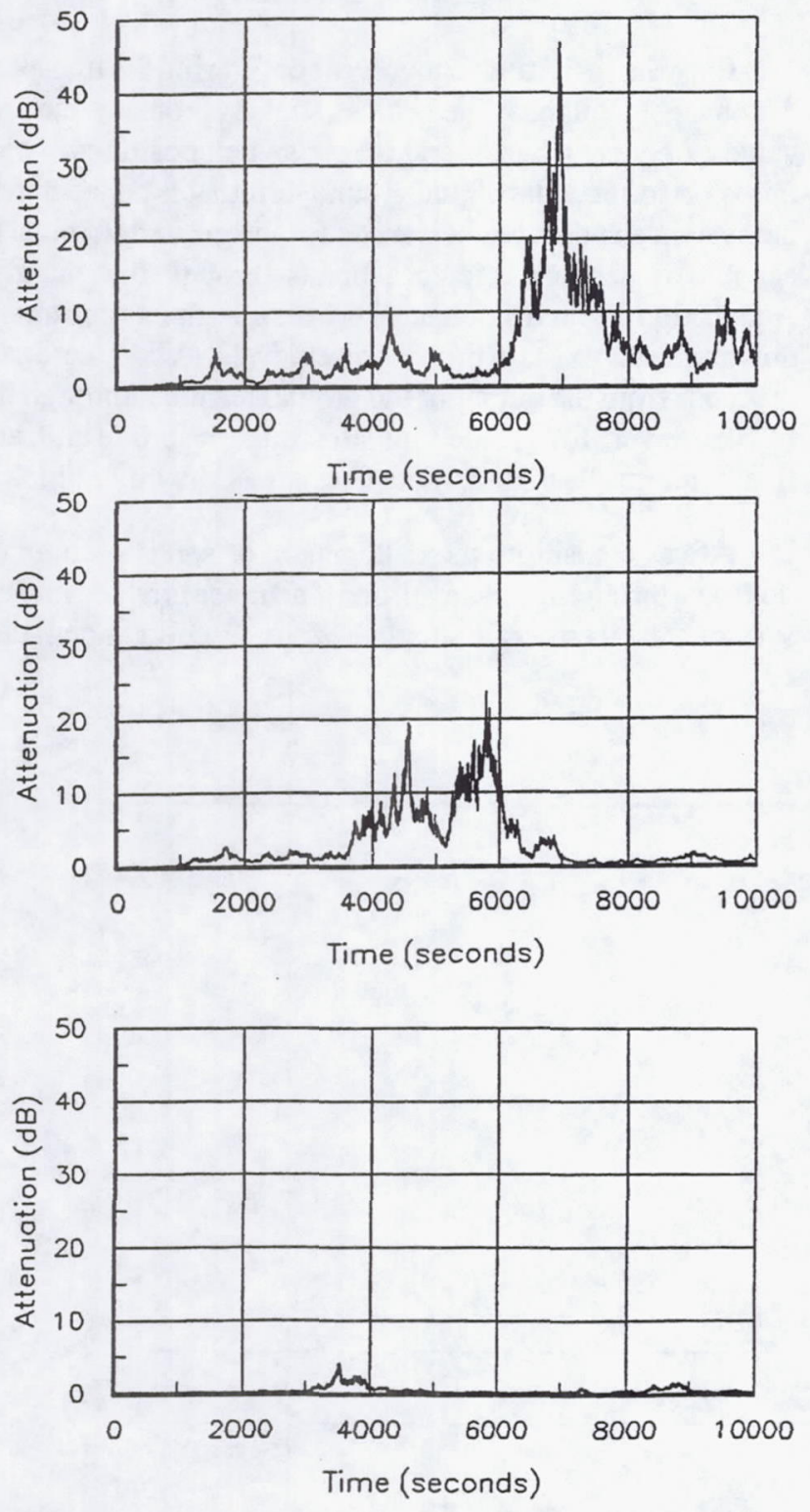

Figure 7.-Dowlink rain fade profiles. tics. ${ }^{4}$ The rain models are stored in software and used to control a programmable power supply which in turn controls the PIN diode rain fade simulator.

Prior to each computer simulation, mathematical rain models require the operator to input various geographic variables, such as mean attenuation, standard deviation of attenuation and temporal attenuation factor. The simulation will calculate the predicted rain attenuation every second, and then translate the attenuation to the proper voltage required by the PIN diode attenuator. Since the rain models are stochastic, every simulation will yield a different profile.

Rain fades are simulated by changing the voltage applied to the PIN diode attenuator in $1 \mathrm{sec}$ increments. The rain fade simulation runs for $10000 \mathrm{sec}$, which corresponds only to rain periods and does not account for clear sky conditions. Figure 7 shows the rain fade profiles for three different cities. Notice that Tampa has the worst rain fade profile due to its tropical typerain. Portland has the least detrimental rain due to its characteristic drizzle whereas Cleveland exhibits properties of both. These attenuation values are due only to the effects of rain, and all other free space and atmospheric losses must be added to the rain losses.

\section{Rain Fade Sense Subsystem}

This subsystem consists of a coaxial directional coupler used to sample the downlink power after the FCS conversion and prior to the demodulator. The power could be sampled before the downconversion at $20 \mathrm{GHz}$, but the interface required for the peak power meter sensor is more practically implemented at the ground terminal IF of $3.375 \mathrm{GHz}$. The coupled power is directed to a peak power meter which in turn produces an analog output voltage proportional to the input RF power.

The peak power meter is capable of measuring power pulses as small as $0.25 \mu \mathrm{sec}$, with a frequency range up to $26.5 \mathrm{GHz}$. A peak power meter, as opposed to a continuous power meter, is necessary for this application due to the many different burst densities used. A continuous power meter will show $\mathrm{a} 3 \mathrm{~dB}$ reduction in power for each halving of the data rate, but the peak power in each burst is the major concern, and this power is the same independent of burst density.

The analog power out of rain fade sense subsystem is amplified and attenuated to assure that the peak power meter sensor input is in the best dynamic range for accurate measurements. Care must be taken in the design of this subsystem to insure that the amplifier is always operating in the linear region so as not to mask power changes by the limiting effects of a saturated amplifier or an amplifier nearing compression.

The threshold detector outputs two bits of digital information in response to the analog voltage-received from the peak powermeter. The two bits indicate either a normal, low, or a high power condition. These conditions can be tuned by adjustable resistors to yield specific thresholds. The two bits of information are then sent to the computer in the MCT ground terminal for processing. 


\section{Computer Systems}

There are four main computer systems involved in the rain fade experiment. Following will be a brief description of each computer system and their functions.

The Experiment Control and Monitor (EC\&M) computer, a Concurrent 3240 minicomputer, is exactly as its name implies. It is responsible for controlling and monitoring the experiment, which involves obtaining and storing data in real-time as well as controlling various IEEE 488 and RS-232 devices. For example, the programmable attenuators of the noise insertion unit that are responsible for incrementing the signal to noise ratio in $1 \mathrm{~dB}$ increments are controlled by an IEEE 488 interface. Readings from power meters are also sent to the EC\&M computer. Using this information, a signal to noise ratio can be calculated. The Master Control Terminal computer, described below, and the data generators/checkers are commanded via RS-232 connections. Bit error rate figures are calculated from the data received from the data checkers.

A Concurrent 3260 minicomputer serves as the Network Control Computer (NCC). The NCC is interfaced to the Digital Routing Processor (DRP) and also connects to the Master Control Terminal (MCT) computer. The NCC is responsible for managing the SS-TDMA network. This includes, but is not limited to administering burst assignments, processing requests for network access and power augmentation (orderwires), maintaining acquisition and frame synchronization, and configuring the IF switch matrix.

The Master Control Terminal (MCT) consists of a 68000 based microprocessor used to acknowledge other ground terminals in the system and receive network access requests from users via orderwires. The MCT microprocessor receives the orderwires through the same path RF used for data transmission, organizes (i.e., discardsinvalid and duplicate requests) and sends them to the NCC. Next, the NCC deciphers the orderwires and informs the MCT of the required action by creation of a reference burst. The MCT notifies the users whether their requests were accepted or denied.

The Digital Routing Processor (DRP) is a satellite based computer/controller that receives commands from the NCC and controls several satellite components. The first component the DRP controls is the pre-TWT attenuator to protect against a TWT input overload. Subsequently, the DRP regulates the power processing unit (PPU) which adjusts the TWT power mode changes. Finally, the DRP must also control the matrix switch crosspoints, but for simplicity and to prevent introducing additional variables, the matrix switch will remain static in one specific path during this experiment.

\section{System Operation}

Experiment initiation involves configuring the transponder channel to all baseline settings such as proper attenuator values, frequency bands, and matrix switch positions. Once this is performed, the digital ground terminal is connected to the system and an attempt is made for the ground terminal to acquire by sending reference bursts through the channel. Acquisition through the channel proves a complete uplink and downlink path exists at proper power levels. A complete path proceeds from the output of the ground terminal through the FCS, receiver, TWT, fade simulator, noise insertion unit, and finally returns back to the data checker as shown in Fig. 2 .

After acquisition, the data generator sends pseudorandom data through the channel, and if no noise is added into the system, the bit error rate should be zero. Pseudorandom data

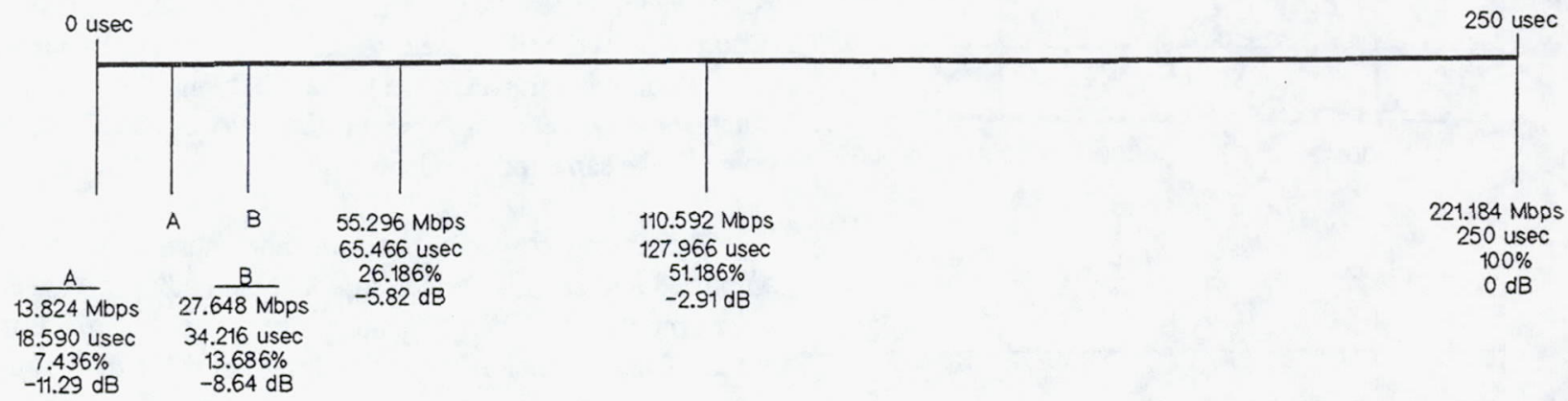

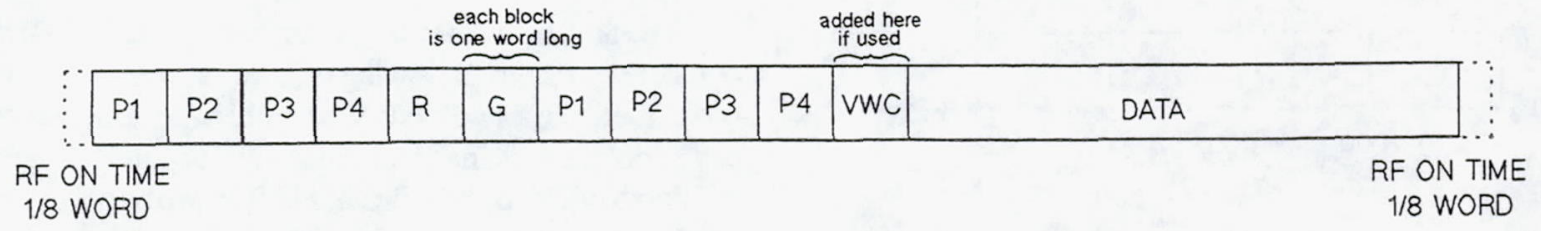

Figure 8.-Digital burst structure. 


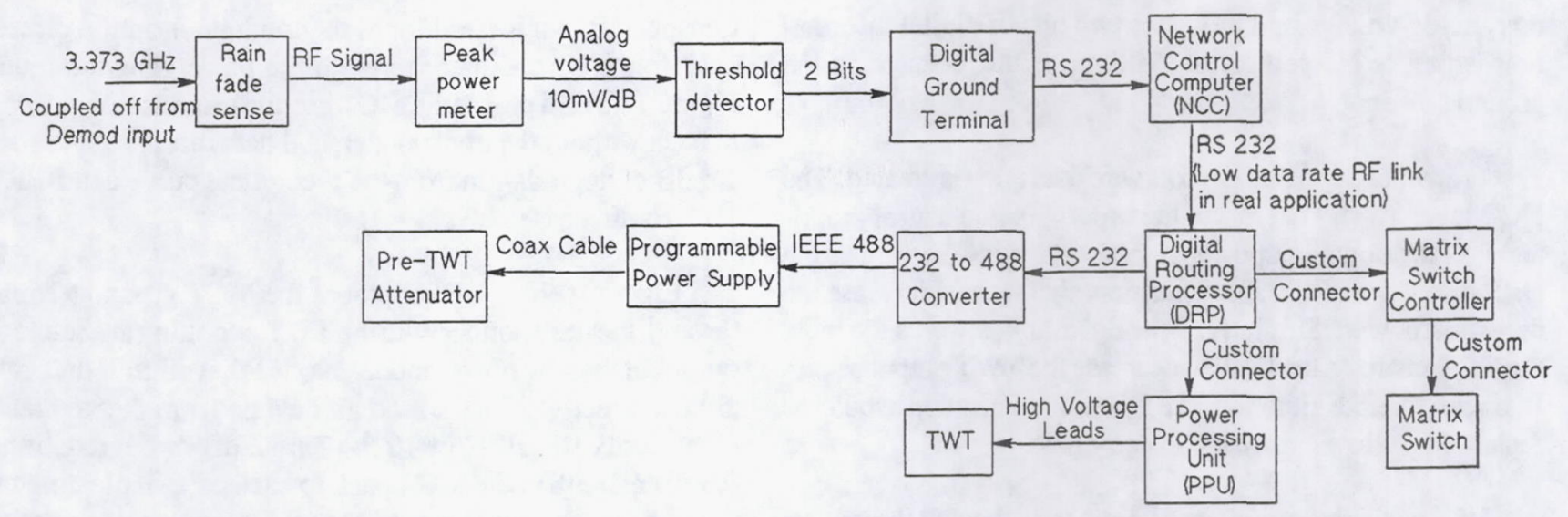

Figure 9.-Rain fade detection/counteraction under network control.

refers to a long repeatable data pattern that resembles random data, but the data checker has prior knowledge of the sequence during error calculations.

An initial ground terminal receive $\mathrm{E}_{\mathrm{b}} / \mathrm{N}_{0}$ of approximately $19 \mathrm{~dB}$ is set, which yields a system just on the verge of running error free, or at least with a BER better than $1.0 \times 10^{-8}$. Any decrease in the received power (as from a rain fade) will result in the datachecker accumulating approximately one error every 1 or $2 \mathrm{sec}$. The initial $19 \mathrm{~dB}$ of $\mathrm{E}_{\mathrm{b}} / \mathrm{N}_{0}$ is not intentionally altered by the noise generation unit, but will change due to rain fades lowering the received signal power.

A Burst Density Power Correction Factor (BDPCF) has been created to aid in the $\mathrm{E}_{\mathrm{b}} / \mathrm{N}_{0}$ calculation by eliminating the need for a low level peak power meter operating in themicrowatt region. All burst densities, regardless of duty cycle, should have the same peak power during the on time. A continuous power meter using a typical thermistor sensor will not measure each burst the same, since each halving in duty cycle will yield a $3 \mathrm{~dB}$ loss in measured power. The BDPCF adds to the continuous power meter readings and yields readings a peak power meter would produce. Figure 8 shows the $250 \mu \mathrm{sec}$ burst division pattern used to create the four burst rates. The lower figure in Figure 8 shows a diagram of a single data burst. The "P" block refers to the preamble, the " $R$ " refers to the reference burst, and the " $G$ " refers to the guard time. Table 1 shows the BDPCF for each data rate, along with other burst division data. Each halving of the data rate does not produce exactly a $3 \mathrm{~dB}$ reduction in power due to the overhead bits of the reference bursts and preamble.

The next step is to initiate a rain fade profile from the EC\&M computer while data is passing through the channel. Some time will pass before the Tampa rain profile produces enough attenuation to cause the threshold detector to detect a threshold crossing. Thus, the Tampa profile was started at $3250 \mathrm{sec}$ in Fig. 7(a) in order to speed up the first threshold crossing. This starting point was chosen since the system must counteract a $6 \mathrm{~dB}$ and then a $10 \mathrm{~dB}$ fade in a short period of time.

In this experiment, the thresholds have been fine tuned to allow just several errors to occur before the threshold detector indicates a low power mode. In a real satellite application, the thresholds would be set to change TWT modes based on the acceptable bit error rate performance for each particular application.

Figure 9 shows the path for rain fade counteraction under network computer control. The rain fade sense subsystem senses the downlink power at the input to the demodulator and sends this power to the peak power meter which produces a proportional analog voltage. The threshold detector samples

Table 1.-BDPCF for Each Data Rate

\begin{tabular}{|c|c|c|c|c|c|c|c|}
\hline $\begin{array}{c}\text { Data } \\
\text { rate, } \\
\text { Mbps }\end{array}$ & $\begin{array}{c}\text { Words } \\
\text { per } \\
\text { frame }\end{array}$ & $\begin{array}{c}\text { Burst } \\
\text { length, } \\
\text { words }\end{array}$ & $\begin{array}{c}\text { Burst } \\
\text { length, } \\
\mu \mathrm{sec}\end{array}$ & $\begin{array}{c}\text { RF on } \\
\text { time } \\
\mu \mathrm{sec}\end{array}$ & $\begin{array}{c}\text { Total } \\
\text { burst } \\
\text { length, } \\
\mu \mathrm{sec}\end{array}$ & $\begin{array}{c}\text { Total } \\
\text { burst } \\
\text { length, } \\
\text { percent } \\
\text { of frame }\end{array}$ & $\begin{array}{c}\text { BDPCF, } \\
\mathrm{dB}\end{array}$ \\
\hline 13.824 & 54 & 64 & 18.518 & 0.07234 & 18.590 & 7.436 & 11.29 \\
27.648 & 108 & 118 & 34.144 & $\downarrow$ & 34.216 & 13.686 & 8.64 \\
55.296 & 216 & 226 & 65.394 & $\downarrow$ & 65.466 & 26.186 & 5.82 \\
110.592 & 432 & 442 & 127.894 & $\downarrow$ & 127.966 & 51.186 & 2.91 \\
\hline
\end{tabular}


this analog voltage and produces two bits of digital information which is relayed to the 6809 computer located in the ground terminal.

From these two bits an orderwire data burst is created. The NCC receives and deciphers the orderwire and acts appropriately. If the downlink power is too low, the NCC instructs the DRP through an RS-232 connection, that it must increase the downlink power. Similarly, if the downlink power is too high, the NCC instructs the DRP to decrease the TWT output power. In a real satellite situation, the RS-232 connection would be replaced by a low data rate telemetry link.

When a power change is invoked, the DRP simultaneously adjusts the attenuation prior to the TWT to prevent a possible overdrive condition. After a rain fade event is counteracted by the method just described, the NCC returns to monitoring for power augmentation orderwires. Upon receipt of another orderwire burst, the entire process is repeated.

\section{Experiment Results}

An important test which must precede any rain fade experiment deals with the ability of the TWT to change power modes while simultaneously transmitting bursted TDMA data. The TWT must have the ability to change power modes through control anode voltage adjustments, and not induce any additional bit errors. If the TWT proves to add errors or causes the demodulator to lose lock, many problems would result, and a method of synchronizing power mode changes to data burst null time would have to be designed.

Experimentsperformed for each of the four data rates (13.8, $27.5,55,110 \mathrm{Mbps}$ ) changed TWT power modes in the following order $(\mathrm{L}, \mathrm{M}, \mathrm{H}, \mathrm{M}, \mathrm{L}, \mathrm{M}, \mathrm{L})$ where the letters denote low, medium and high modes. Each of the four data rates accumulated zero bit errors during the entire data run, even during the exact time of the TWT power mode transitions. These results ensure that demodulator synchronization is not altered during power mode changes, and therefore confirms no additional data errors are induced by TWT mode transitions.

Another test which must be performed prior to the rain fade experiment deals with the bit error characteristics of each TWT power mode. Ideally, the TWT should perform the same in terms of bit error rate for each of the three power modes. If this were not the case, a power mode increase may not improve the error rate at all, and may even degrade the error rate more than the lower power mode. All the BER curves shown were created by the bit error control program on the EC\&M computer which automatically increments the $\mathrm{E}_{\mathrm{b}} \mathrm{N}_{0}$ through remote control of the noise generation unit, while the data checker simultaneously counts the number of bit errors.

BER curves were plotted for each of the three power modes using all four burst densities. A ground terminal back to back curve was also plotted, which does not use the transponder, but instead loops the modulator output directly back to the demodulator through the noise generation unit. Figure 10(a) is a plot of the MCT ground terminal looped back to back without the transponder. All data rates provide about $1.5 \mathrm{~dB}$ of degradation from the theoretical curve at a BER of $10^{-6}$ for this case.

Figure 10(b) is a BER plot of the MCT ground terminal through the transponder with the TWT operating at a saturated output in the low power mode. Notice that all four data rates almost directly coincide, and all deviate from theoretical by about $2 \mathrm{~dB}$ at a BER of $10^{-6}$. This $2 \mathrm{~dB}$ of degradation is $0.5 \mathrm{~dB}$ more than the MCT back to back case, indicating that the transponder contributes about $0.5 \mathrm{~dB}$ more degradation. Figure $10(c)$ is another MCT ground terminal curve, but this time the TWT is in medium mode. Again, all four burst densities coincide, and all show about $2 \mathrm{~dB}$ degradation from theoretical at a BER of $10^{-6}$. The same is true for the TWT high mode case in Fig. 10(d). The results indicate that the BER performance of all three TWT power modes is nearly identical, and that all burst densities perform the same if the demodulator input power is kept constant.

\section{Rain Simulation/Counteraction Under Network Control}

The operation of the complete rain fade system outlined in Figs. 2 and 9 is the ultimate test of the entire rain fade simulation/ counteraction system since it ties together all aspects of the project: data transmission, TWT power adjustments, full computer control, and rain fade profiles. The system is totally automated, and once a rain fade profile is initiated, all TWT power mode adjustments and any pre-TWT power conditioning is controlled by the NCC and DRP computers.

Figure 11 shows the errors accumulated both with and without TWT power control during a $20 \mathrm{~min}$ Tampa rain fade. Notice in the network disabled case as the rain attenuation starts to increase at around $3600 \mathrm{sec}$, the errors begin to increment and level off at 680 errors. No additional errors are accumulated during the period between 3610 and 4210 since the fade profile during this period drops below $5 \mathrm{~dB}$ for most of the interval. At around 4220 , the fade begins to build to over $10 \mathrm{~dB}$, at which time the number of errors dramatically increases to over 22 million with a corresponding accrued BER greater than $10^{-4}$. The number of errors then remains relatively constant as the fade begins to fall back to $4 \mathrm{~dB}$.

The number of errors accumulated with the network operational is also displayed in Fig. 11. Just as for the network disabled case, the operational case begins to accumulate errors as the fade approaches the $6 \mathrm{~dB}$ apex. Notice that only five errors accumulate during the 3610 to 4270 time interval. The reason for this low number of errors is due to a TWT low to medium power transition which occurred at $3600 \mathrm{sec}$ and overpowered the $6 \mathrm{~dB}$ fade. The TWT remained in medium mode until $4310 \mathrm{sec}$ at which time it changed to high mode in response to the building $10 \mathrm{~dB}$ fade. Notice that the $10 \mathrm{~dB}$ fade only forced a total of 160 errors to accumulate as opposed to 


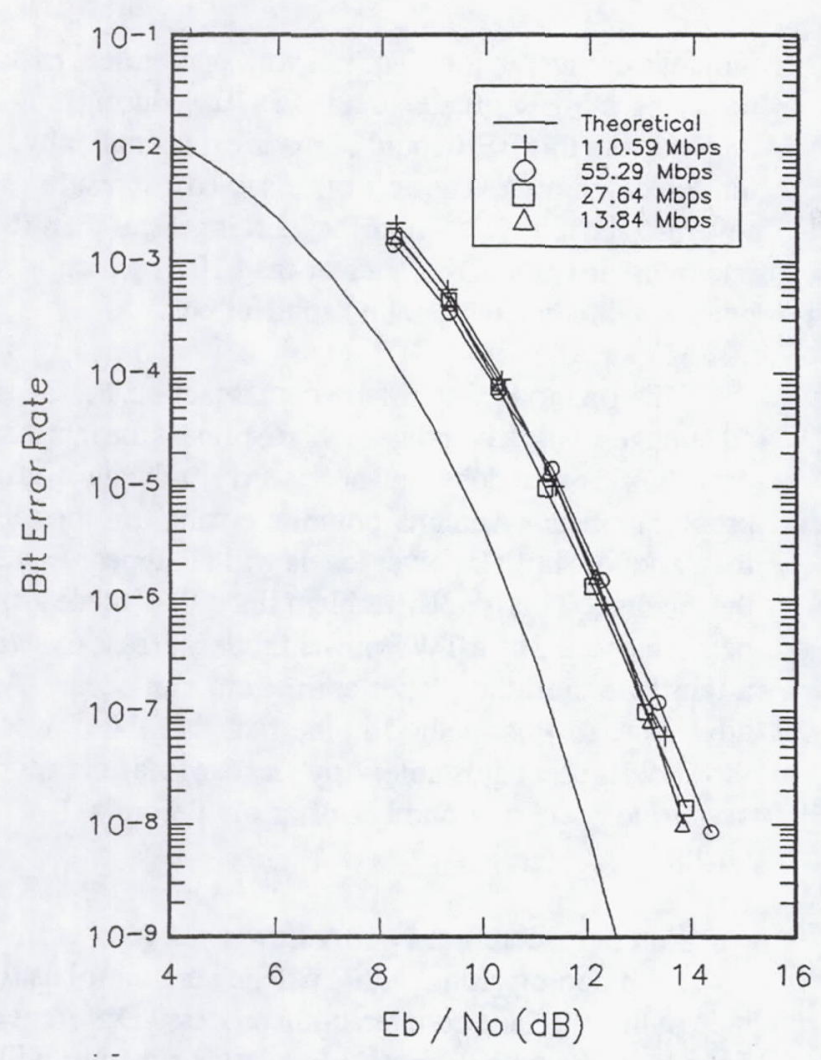

(a) MCT ground terminal back to back.

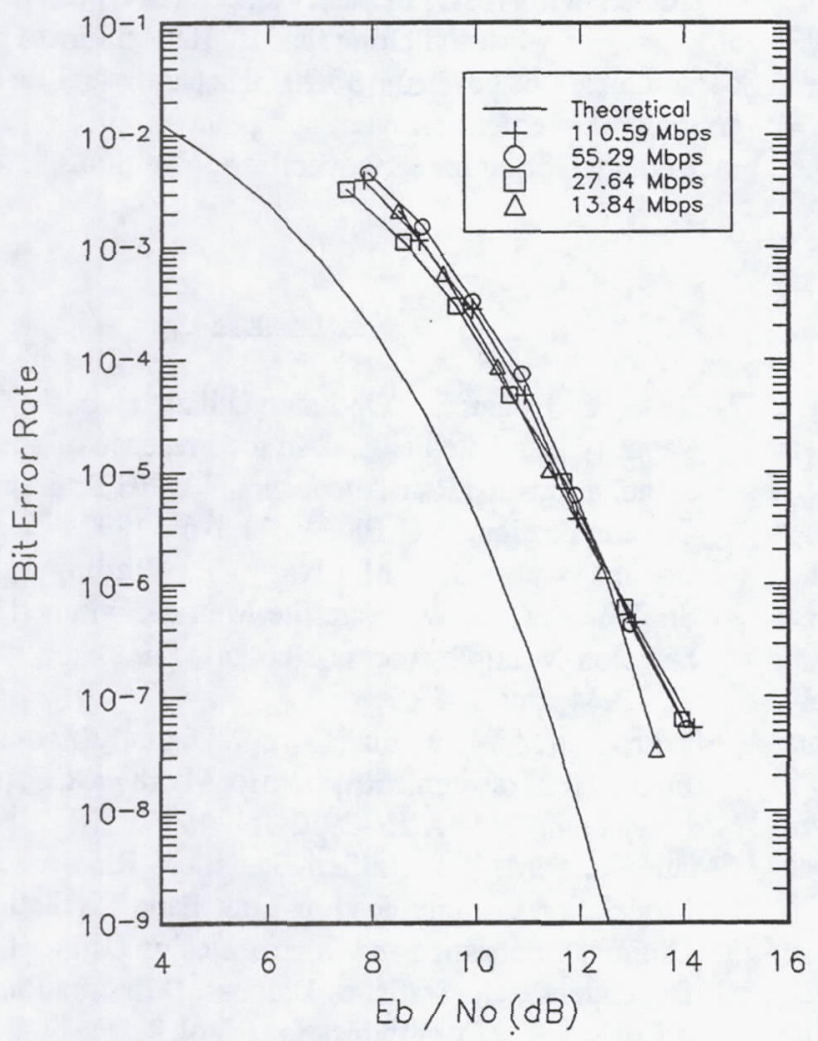

(b) MCT ground terminal with TWT low mode saturated.

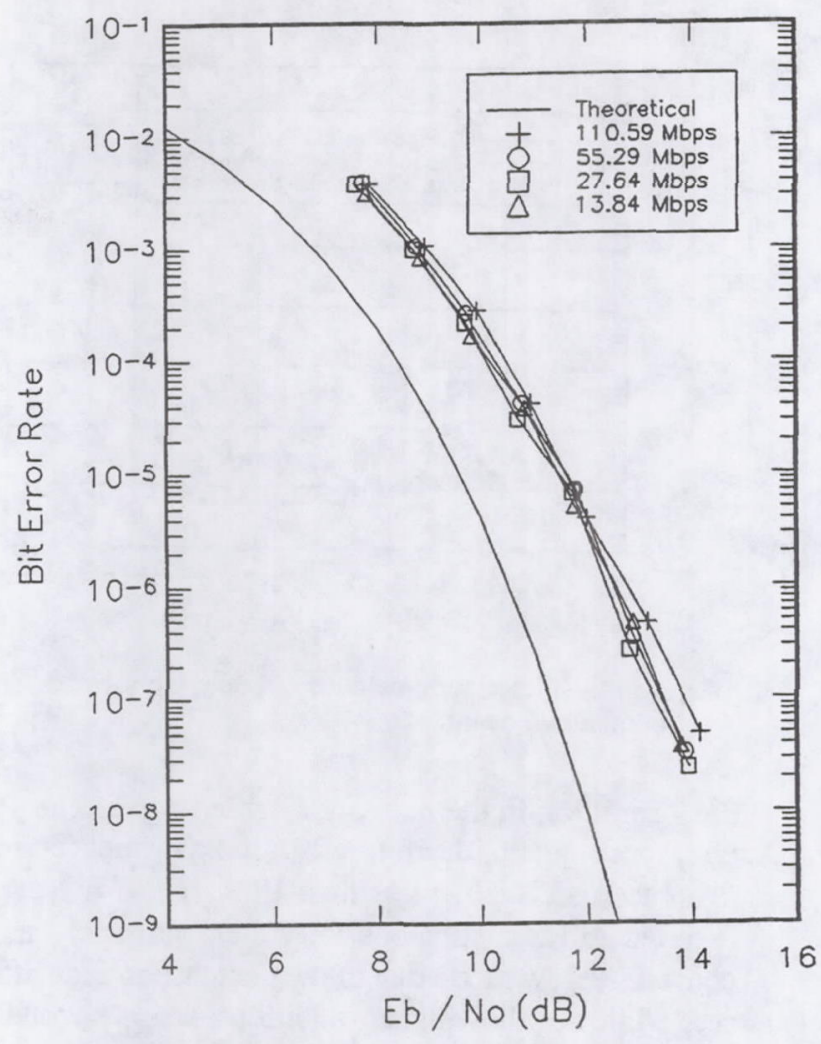

(c) MCT ground terminal with TWT medium mode saturated.

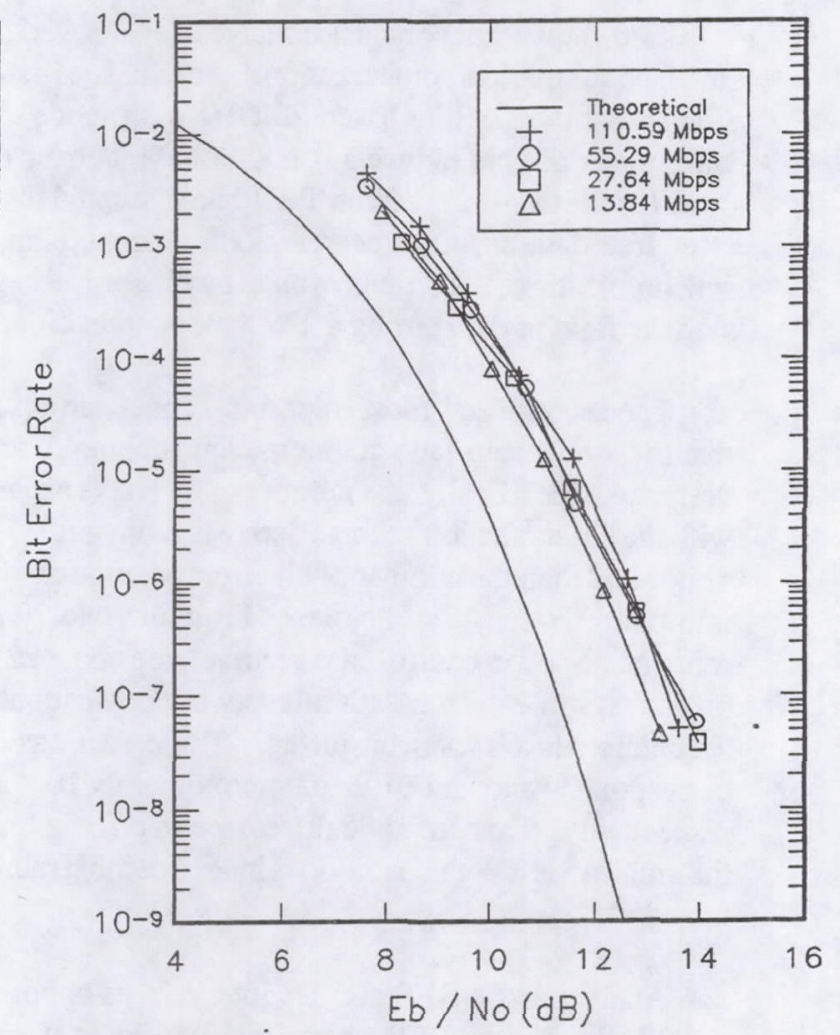

(d) MCT ground terminal with TWT high mode saturated.

Figure 10.-Bit erro: rate surves. 




Figure 11.-Errors accumulated with and without power contro: during a rain event.

the 22 million in the network disabled case. The 160 total errors accumulated during the 20 min experiment corresponds to an accrued BER of less than $10^{-8}$. The TWT eventually reverted to medium mode, and then ultimately to energy conserving low mode due to the decrease in fade after 4270 sec, while not allowing any additional errors beyond the 160 already present.

\section{Conclusions}

Two significant conclusions have been derived from the rain fade simulation/counteraction research. The first conclusion is that the satellite based $20 \mathrm{GHz}$ multimode traveling wave tube does not induce any additional bit errors during a power mode transition. If the TWT had disrupted the flow of error free data during a power mode change, complicated synchronization algorithms would have been necessary to stop the flow of data during a TWT mode transition.

The second, and most important, conclusion is the fact that the whole rain fade counteraction scheme functions as designed. Specifically, the number of bit errors accumulated with the counteraction scheme active is many orders of magnitude less than the number of bit errors accumulated with no rain fade counteraction invoked. From the two 20 min test runs, the no power control case accumulated about 22100000 bit errors, while the run with full network power control only accumulated 160 total bit errors. These two error counts correspond to accrued BERs of approximately $10^{-4}$ and $10^{-8}$ respectively. This dramatic difference in error count proves the multimode TWT scheme does indeed combat rain induced bit errors.

Analyzing the BER at $5 \mathrm{sec}$ intervals, as opposed to the full 20 min accrued BER, shows the significant effects of the rain fade counteraction. The network operational case never produces a BER greater than $2.5 \times 10^{-7}$ during any $5 \mathrm{sec}$ interval, and this BER could be made even smaller by threshold detector tuning. The network disabled test results produce several $5 \mathrm{sec}$ intervals where the BER is greater than $10^{-3}$ and one interval with a BER greater than $10^{-2}$, which would be unacceptable in most satellite applications.

This method of rain fade counteraction has additional advantages. One advantage of this method is the fact that alow downlink power does not necessarily indicate a rain fade problem exists. Antenna pointing errors, transponder gain loss, and low uplink power levels will all appear as a fade at the receiver. This is not a problem since all of these losses can be counteracted by a TWT power mode increase exactly as in a rain fade until the proper corrections can occur. Another advantage to this method is the fact that the power mode threshold is user adjustable. Any user can fine tune a particular system to yield any number of errors before a TWT mode change.

Future research at NASA Lewis plans to examine the effects of coding along with TWT power control as the next step in the rain fade counteraction process. Different coding formats along with different modulation schemes will determine the most rain tolerant satellite downlink combination. Scintillation, very rapid changes in path attenuation, can also be studied in the ASC laboratory since the PIN diode attenuators have very fast switching speeds. If the future of satellite communications lies in the $30 / 20 \mathrm{GHz}$ band, satellite designers must implement efficient and practical methods of rain fade counteraction for each specific application.

\section{$\underline{\text { References }}$}

${ }^{1}$ Filip, M. and Vilar, E., "Optimum Utilization of the Channel Capacity of a Satellite Link in the Presence of Amplitude Scintillations and Rain Attenuation," IEEE Transactions on Communications, Vol. 38, No. 11, Nov. 1990.

${ }^{2}$ Ivancic, W., Andro, M., Nagy, L., Budinger, J. and Shalkhauser, M.W.,"Satellite-Matrix-Switched, TimeDivision-Multiple-Access Network Simulator," NASA TP-2944, Oct. 1989.

${ }^{3}$ Shalkhauser, M.W. and Budinger, J., "Digitally Modulated Bit Error Rate Measurement System for Microwave Component Evaluation,"NASA TP-2912, July 1989.

${ }^{4}$ Manning, R.M., "A Unified Statistical Rain-Attenuation Model For Communication Link Fade Predictions and Optimal Stochastic Fade Control Design Using a Location Dependent Rain-Statistics Database," International Journal of Satellite Communications, Vol. 8, No. 11-30, 1990. 
Public reporting burden for this collection of information is estimated to average 1 hour per response, including the time for reviewing instructions, searching existing data sources, gathering and maintaining the data needed, and completing and reviewing the collection of information. Send comments regarding this burden estimate or any other aspect of this collection of information, including suggestions for reducing this burden, to Washington Headquarters Services, Directorate for In Davis Highway, Suite 1204, Arlington, VA 22202-4302, and to the Office of Management and Budget, Paperwork Reduction Project (0704-0188), Washington, DC 20503.

\begin{tabular}{|l|l|l}
\hline 1. AGENCY USE ONLY (Leave blank) & $\begin{array}{c}\text { 2. REPORT DATE } \\
\text { December } 1993\end{array}$ & $\begin{array}{r}\text { 3. REPORT TYPE AND DATES COVERED } \\
\text { Final Contractor Report }\end{array}$
\end{tabular}

\section{TITLE AND SUBTITLE}

5. FUNDING NUMBERS

A Laboratory System for the Investigation of Rain Fade Compensation Techniques for Ka-Band Satellites

\section{AUTHOR(S)}

James S. Svoboda and Brian A. Kachmar

Sverdrup Technology, Inc.

Lewis Research Center Group

2001 Aerospace Parkway

E-8186

Brook Park, Ohio 44142

9. SPONSORING/MONITORING AGENCY NAME(S) AND ADDRESS(ES)

10. SPONSORING/MONITORING

National Aeronautics and Space Administration

Lewis Research Center

Cleveland, Ohio 44135-3191

NASA CR-191200

AIAA-94-0917

\section{SUPPLEMENTARY NOTES}

Prepared for the 15th International Communications Satellite Systems Conference, San Diego, California, February 28-March 4, 1994. James S.

Svoboda, Sverdrup Technology, Inc. (work funded by NASA Contract NAS3-25266); and Brian A. Kachmar, Analex Corporation, 3001 Aerospace

Parkway, Brook Park, Ohio 44142 (work funded by NASA Contract NAS3-25776). Project Manager, Godfrey Anzic, Space Electronics Division, (216) 433-3570.

12a. DISTRIBUTION/AVAILABILITY STATEMENT

Unclassified - Unlimited

Subject Category 33

13. ABSTRACT (Maximum 200 words)

The design and performance of a rain fade simulation/counteraction system on a laboratory simulated $30 / 20 \mathrm{GHz}$, time division multiple access (TDMA) satellite communications testbed is evaluated. Severe rain attenuation of electromagnetic radiation at $30 / 20 \mathrm{GHz}$ occurs due to the carrier wavelength approaching the water droplet size. Rain in the downlink path lowers the signal power present at the receiver, resulting in a higher number of bit errors induced in the digital ground terminal. The laboratory simulation performed at NASA Lewis Research Center uses a programmable PIN diode attenuator to simulate $20 \mathrm{GHz}$ satellite downlink geographic rain fade profiles. A computer based network control system monitors the downlink power and informs the network of any power threshold violations, which then prompts the network to issue commands that temporarily increase the gain of the satellite based traveling wave tube (TWT) amplifier. After the rain subsides, the network returns the TWT to the normal energy conserving power mode. Bit error rate (BER) data taken at the receiving ground terminal serves as a measure of the severity of rain degradation, and also evaluates the extent to which the network can improve the faded channel.

\begin{tabular}{|c|c|}
\hline \multicolumn{2}{|l|}{ 14. SUBJECT TERMS } \\
\hline $\begin{array}{l}\text { 17. SECURITY CLASSIFICATION } \\
\text { OF REPORT } \\
\text { Unclassified }\end{array}$ & $\begin{array}{l}\text { 18. SECURITY CLASSIFICATION } \\
\text { OF THIS PAGE } \\
\text { Unclassified }\end{array}$ \\
\hline
\end{tabular}

